\title{
Cascaded H-Bridge Multilevel Converter Multistring Topology for Large Scale Photovoltaic Systems
}

\author{
S. Rivera**, S. Kouro*, B. Wu*, J. I. Leon***, J. Rodríguez**, L. G. Franquelo*** \\ *Department of Electrical and Computer Engineering, Ryerson University, Toronto, Ontario, Canada \\ **Electronics Engineering Department, Universidad Técnica Federico Santa María, Valparaíso, Chile \\ ***Electronics Engineering Department, University of Seville, Spain
}

\begin{abstract}
Large scale grid connected photovoltaic (PV) energy conversion systems have reached the megawatt level. This imposes new challenges on existing grid interface converter topologies and opens new opportunities to be explored. In this paper a new medium voltage multilevel-multistring configuration is introduced based on a three-phase cascaded H-bridge (CHB) converter and multiple string dc-dc converters. The proposed configuration enables a large increase of the total capacity of the PV system, while improving power quality and efficiency. The converter structure is very flexible and modular since it decouples the grid converter from the PV string converter, which allows to accomplish independent control goals.

The main challenge of the proposed configuration is to handle the inherent power imbalances that occur not only between the different cells of one phase of the converter but also between the three phases. The control strategy to deal with these imbalances is also introduced in this paper. Simulation results of a 7-level CHB for a multistring PV system are presented to validate the proposed topology and control method.
\end{abstract}

\section{INTRODUCTION}

Grid connected solar photovoltaic energy conversion systems are the fastest growing renewable energy source in installed capacity in the last 5 years. In fact, it has increased an annual average of $60 \%$ per year between 2004 and 2009 (7 GW installed in 2009 only, with a total of $21 \mathrm{GW}$ installed capacity world wide) [1]. Large-scale PV utility plants range from $200 \mathrm{~kW}$ up to almost $100 \mathrm{MW}$ in total capacity (the largest is of $97 \mathrm{MW}$ in Sarnia, ON, Canada). Currently there are more than 150 utility-scale PV plants over 10 MW [2]. There is a clear global trend to increase the capacity and quantity of utility-scale PV plants.

Currently large-scale PV plants are interfaced by two type of power converter configurations: the centralized topology and the multistring topology [3]. The centralized topology is characterized by a large amount of PV modules in series to reach the desired PV string voltage. Several of these strings are then paralleled to reach the total power level of the PV system. The dc power is interfaced to the utility by a centralized gridtied inverter, most likely a three-phase 2-level voltage source inverter. Isolation, if required, is usually provided by a low frequency transformer at the ac side. The advantage of this configuration is the simplicity of the structure and control (only one converter) and reduced cost. The main disadvantage is the lower power output due to a single maximum power point tracking (MPPT) for the whole plant, which is affected by module mismatch and partial shading.
On the other hand, the multistring concept [4] also uses a centralized grid tied inverter, but has a distributed dc-bus in which each string is connected through a dc-dc converter. Commonly these are boost converters, if isolation is provided at the ac side, or a high frequency isolated dc-dc converters (like a flyback or push-pull converter), if isolation is required at dc side [5]. The main advantage of the multistring concept is the increased modularity, allowing to combine different types of modules and even dc-dc string converters. It also decouples the grid converter control from the PV string control, which allows independent MPPT tracking of each string, increasing the power output. The main disadvantage is the higher cost and topology complexity of having additional power converters, sensors, and control systems. Nevertheless, the higher conversion efficiency has proven to be a superior advantage in long term operation, hence it is considered the state of the art configuration today.

Both configurations commonly operate with the centralized inverter at low voltage $(690 \mathrm{~V})$, which given current limitations of semiconductors, allows a power rating of up to $0.7 \mathrm{MW}$ without paralleling devices or converters. This imposes a severe limitation for large scale PV plants (in the megawatt range), where several centralized converters are needed to interface the power. The converters can be used as separate centralized topologies dividing the PV plant in subsystems, or connected in parallel as a single one to handle all the power of the PV plant.

The trend of megawatt range PV plants will demand higher power ratings for the central grid tied converter, and traditional two level voltage source converters (2L-VSC) topologies will not be able to fulfill power rating, power quality and efficiency requirements. Moreover, more demanding grid codes could apply to these systems as happens today with wind energy conversion systems [6], pushing further the limits of the $2 \mathrm{~L}$ VSC. The use of several 2-level converters also means more power electronics, control systems, sensors, filters, size and cost compared to using a single medium-voltage high-power converter.

Medium voltage converters have been proposed recently for grid connected PV systems [7]-[15]. Most of these proposals are based on the 3-level NPC multilevel converter, and the single-phase cascaded H-bridge multilevel converter. The NPC topology can be commercially found up to several tens of megawatt (up to 40MVA) and typically connected to $3.3 \mathrm{kV}$ 
and $4.16 \mathrm{kV}$ grids [16]. To fully use the power rating of an NPC converter too many modules need to be connected in series to reach medium voltage, and several more in parallel to reach desired power levels. This issue comes back to the same problem of the centralized topology. An improvement has been made with an NPC multistring approach [15], where the dc-dc stage can help boosting the voltage reducing the number of modules in series. In addition parallel connection is performed with individual strings and their respective dc-dc converters with all the advantages of the multistring concept.

The CHB has particular advantages for PV systems: it provides several dc-links to which connect PV strings, each one with independent MPPT, and it easily reaches medium voltage. Nevertheless, since each H-bridge cell has its independent PV system with its own power point, there is an inherent power imbalance between the cells. If this imbalance is not taken into consideration in the control system or modulation, the dc-link voltages will drift. The dc-link voltage imbalance degrades the power quality introducing voltage distortion at the grid side, and more importantly, represents a hazard for the converter if voltage limits of the capacitors are exceeded. This has been addressed in several ways for single phase systems [12], [14]. To reach higher capacity for large scale plants, three-phase configurations are needed. However, the three-phase CHB for PV system introduces an additional challenge, which is the inherent imbalance between the three phases, since each cell has its own MPPT. This will lead to unbalanced currents, which is not allowed by grid codes.

This paper proposes a compensation method in the modulation stage of the three-phase CHB converter to deal with this imbalance, by shifting the neutral of the reference voltages in such a way the currents are balanced. This is achieved through a weighted zero sequence injection, in which each phase voltage reference is inversely compensated according to the respective imbalance ratio. This acts as a feedforward mechanism correcting the undesired behavior. In addition, to increase the power capacity of the total PV system, the multistring concept is introduced to each dc-link of the CHB. This enables to connect several strings in parallel to each $\mathrm{H}-$ bridge cell, each with its independent MPPT. In this way, very large PV plants can be concentrated into a single CHB (which can be found up to 120MVA [16]), with the benefits of: high power quality, increased efficiency, one control system, one set of sensors, one line filter, etc. The proposed configuration and control system is simulated for a three-phase 7-level CHB (3 cells), which in practice reaches medium voltage level of $3.3 \mathrm{kV}$ for current semiconductor limits. Nevertheless, the configuration and control method can be directly extended for CHBs of any number of levels (the 13 level with 6 cells in series reaches $6.6 \mathrm{kV}$ and together with the 3 -cell version are the most popular [16]).

\section{TOPOLOGY DESCRIPTION}

The three-phase CHB multilevel multistring PV system configuration is illustrated in Fig. 1. The power circuit is composed of three parts: the H-bridge power cells of the $\mathrm{CHB}$,

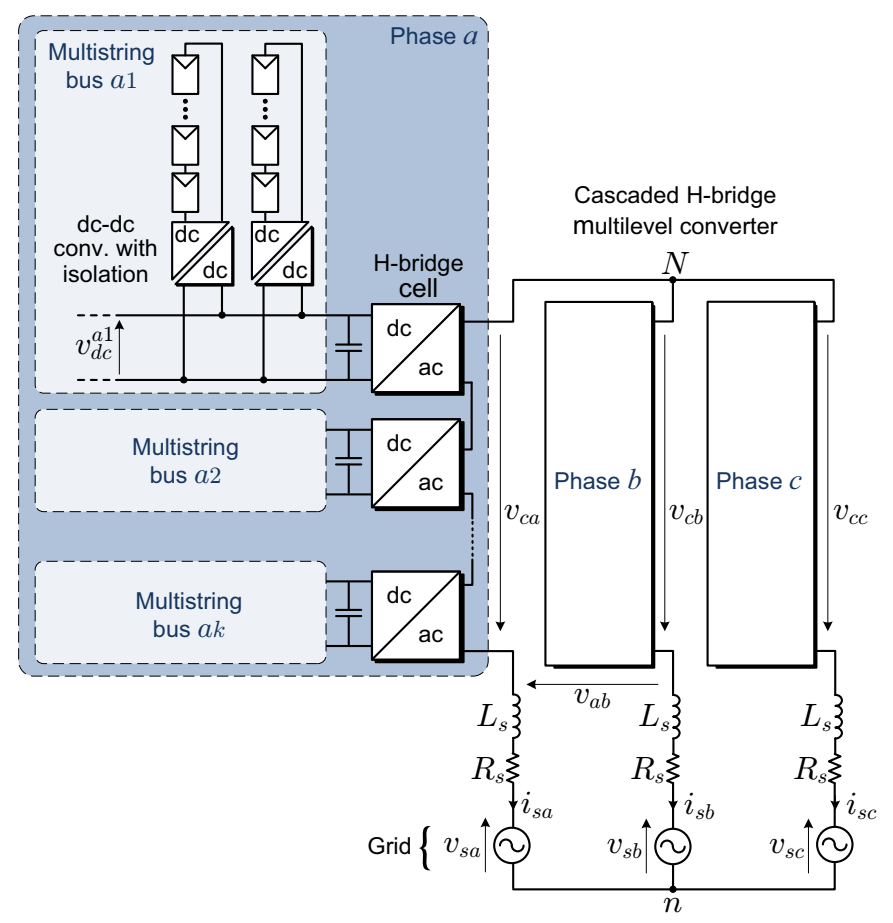

Fig. 1. Proposed cascaded H-bridge multistring PV system configuration.

the PV strings, and the dc-dc converters (with isolation is preferred to ground the strings and reduce risk of hazard). Note only one phase is given in detail due to space limitations.

Usually commercial $\mathrm{CHBs}$ are of three cells per phase $(k=3)$ to reach $3.3 \mathrm{kV}$ or of six cells $(k=6)$ to reach $6.6 \mathrm{kV}$ medium voltage levels. The converter ac voltages $\left(v_{c a}, v_{c b}\right.$ and $\left.v_{c c}\right)$ are made of the sum of the cell output ac voltages and are usually modulated using Phase-Shifted PWM (PS-PWM) to ensure even cell usage [17]. Each cell is modulated with unipolar PWM where the carrier signals are shifted between cells in $\pi / k$ rad to produce the multilevel stepped voltage waveform and optimize power quality. It is common to use low switching frequencies due to the power rating of the system, semiconductor limits, efficiency and heat dissipation requirements. Typical carrier signals for each cell (hence average device switching frequency) are of $500 \mathrm{~Hz}$, which lead to $1 \mathrm{kHz}$ cell output equivalent frequency (due to unipolar PWM) and to $k \mathrm{kHz}$ equivalent total converter output switching frequency due to the multiplicative effect of the phase-shifts of the carriers among cells. This is why the CHB topology produces lower losses compared to several 2-level converters paralleled to reach same power rating, operating at higher switching frequencies to reach same power quality. In addition, the $\mathrm{CHB}$ reaches medium voltage, for which no step up transformer is required (isolation can be obtained in dc-dc stage of the multistrings).

The PV strings are composed by the series connection of several PV modules, to reach a voltage level close to the dclink voltage of one cell of the $\mathrm{CHB}$ converter. The number of modules per string can be different, they can be of different type and interfaced through different dc-dc converters, as long 


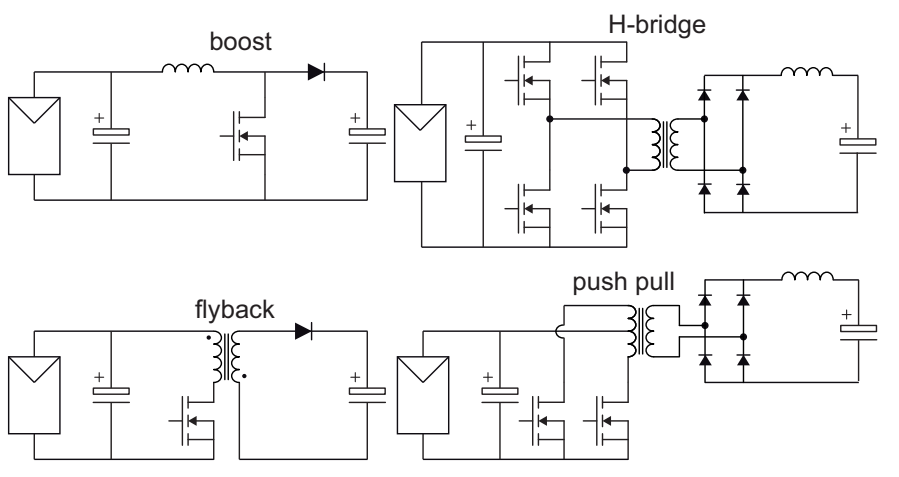

Fig. 2. Different types of dc-dc converter stages for string connection (without isolation in case of the boost converter).

as the dc-converter is capable to boost the voltage up to the dc-link voltage level $\left(v_{d c}^{i j}\right.$, where $i=a, b, c$ stands for the phase, and $j=1,2, \ldots, k$ for the cell number). Several strings can be connected to each dc-bus up to the power rating allowed by each cell. Although the strings are connected in parallel to the dc-bus, they have each independent MPPT thanks to their dedicated dc-dc converter. For this reason the multistring system is very flexible and modular. The control technique proposed in this paper is able to deal with uneven power distribution among the cells and hence it is technically feasible to have different installed capacity in each dc-bus. Nevertheless, it is desirable to design the system as balanced as possible (same installed capacity per dc-bus) and leave the imbalances to mismatch among modules, partial shading and even disconnection of a complete string (or more) per dc-bus. In this way the proper operation and performance of the overall system can be extended for a greater range.

As mentioned before, the dc-dc converters can be of different type. Fig. 2 shows commonly used dc-dc stages for PV multistrings systems [5]. The boost converter is one of the most commonly used where systems without isolation are permitted or where ac side isolation is used. In case of the CHB topology it is recommended to use dc-side isolation, so each string can be grounded to avoid hazard conditions produced by parasitic capacitances of the PV modules. In this work, the main contribution is the analysis and control of the power imbalances of the grid tied converter, and therefore less detail will be given on the dc-dc stage control and topology. For sake of simplicity flyback converters were considered in this work.

\section{Proposed Control Method}

Two types of independent control loops are used to control the multistring topology: one for the grid tied inverter which controls the dc-link voltages and grid currents and the other for the dc-dc stage converters which is used to control the PV string voltage (input dc-side of the dc-dc converter) and adjust it to a desired reference given by the MPPT algorithm. Since the aim of this paper is not a contribution in relation to the dc-dc stage control, classic cascaded current and voltage loops are considered along with the well known perturb and observe (P\&O) algorithm [18], [19]. The following subsections

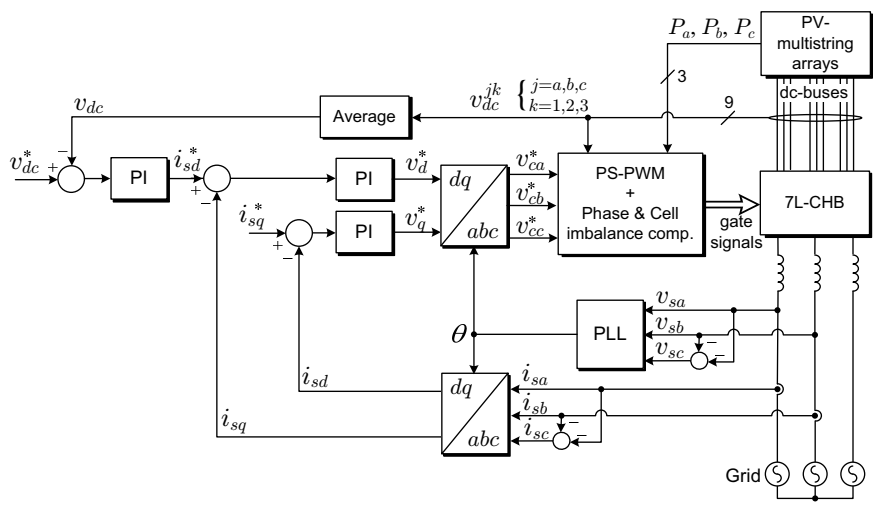

Fig. 3. Voltage oriented control diagram with imbalance compensation for proposed topology.

describe the control method and power imbalance compensation technique for the centralized CHB converter.

\section{A. Voltage oriented control}

Two high performance control methods are considered as main-stream solution for grid tied converters: Voltage Oriented Control (VOC), and Direct Power Control (DPC) [20]. Improved versions of both methods based on the virtual flux concept have been also proposed. In this work the VOC method is considered, mainly because it has a modulation stage embedded in the loop (DPC does not use modulation), which makes dealing with the power imbalance a more approachable task, particularly since it is a multilevel converter.

The modified VOC scheme block diagram is illustrated in Fig. 3. As with classic VOC, there is an outer voltage control loop with an embedded inner current control loop. The outer loop controls the dc-link voltage, and since the $\mathrm{CHB}$ has several dc-links, an average of them is controlled. In this way the total active power needed to control all the dc-link voltages is computed. The distribution of that active power among the different cells is later carried out in the modulation stage thanks to the per-phase and per-cell balancing mechanisms (analyzed later in this paper). The active power reference given by the voltage loop, is proportional to the $i_{s d}$ current component, while the reactive power is proportional to the $i_{s q}$ component. The reactive power reference is usually set to zero, although it can be controlled at different values if required. Both currents are regulated with PI controllers that give the converter reference voltage, which is then converted from $d q$ reference frame to three-phase voltage references. The orientation of the $d q$ transformation, or synchronization, is performed through a PLL with the measured phase voltages of the grid, to ensure unity power factor operation. The threephase grid currents are measured and fed back for the current control loop. The voltage reference given by the current loop is then modulated using phase-shifted PWM. Note that in order to properly control the dc-link voltages of each cell and compensate the inherent power imbalances introduced by the different PV strings, the modulation stage needs to be modified to address these issues. The problem description and solution 
for the power imbalance is discussed in the next sections.

\section{B. Power Imbalance Problem Description}

The power generated by a PV module depends strongly on two factors: solar irradiance and operating temperature of the module. The maximum power point will depend on these two operating conditions which may vary slightly between adjacent modules, but can be quite different between several sectors of large PV plants due to partial shading and module mismatch. Because of this it is very unlikely to have identical power delivered by each PV string to the power cells of the $\mathrm{CHB}$ causing power imbalance among them. Moreover, strings of different size, or interfaced with different dc-dc converters and control systems may further add to the imbalance.

There are two types of power imbalance: per-cell imbalance and per phase imbalance. The first is the power imbalance between the cells of one phase. This means for example that the power processed by each $\mathrm{H}$-bridge of phase $a$ are not equal $\left(P_{a 1} \neq P_{a 2} \neq P_{a 3}\right.$ for a three-cell $\left.\mathrm{CHB}\right)$. The second is the difference of total power processed by each phase of the converter $\left(P_{a} \neq P_{b} \neq P_{c}\right)$. These two type of imbalance affect the control of the CHB converter in two different ways: the per-cell imbalance affects the dc-link control loop making the dc-link voltages from one phase to drift from the reference value, which causes distortion and may harm the converter; the per-phase imbalance affects the current control loop making the grid current unbalanced among each other. The fact that VOC returns a single balanced voltage reference, means that if the power is different among the cells the currents must be imbalanced. This is not allowed by existing grid codes.

\section{Power Imbalance Compensation Method}

The two types of imbalances will be dealt with sequentially: first the per-phase imbalance is compensated generating a new reference voltage for each phase, and later the per-cell imbalance is compensated generating a new reference voltage for each cell.

1) Per-phase imbalance compensation: The motivation behind the per-phase imbalance compensation method lies in the core of the origin of the problem. Since VOC generates the same voltage reference amplitude for each phase, if the power is unbalanced the resulting currents will be unbalanced. The idea then is to unbalance the voltage reference in an inverse way, proportional to the power imbalance, so that the resulting currents are balanced and fulfill grid code requirements. This can also be seen as a sort of feedforward compensation.

Consider the phase voltage equations from the power circuit shown in Fig. 1 at the grid side given by

$$
\begin{aligned}
v_{c a}+v_{N n}-R_{s} i_{s a}-L_{s} \frac{\mathrm{d} i_{s a}}{\mathrm{~d} t}-v_{s a} & =0, \\
v_{c b}+v_{N n}-R_{s} i_{s b}-L_{s} \frac{\mathrm{d} i_{s b}}{\mathrm{~d} t}-v_{s b} & =0, \\
v_{c c}+v_{N n}-R_{s} i_{s c}-L_{s} \frac{\mathrm{d} i_{s c}}{\mathrm{~d} t}-v_{s c} & =0 .
\end{aligned}
$$

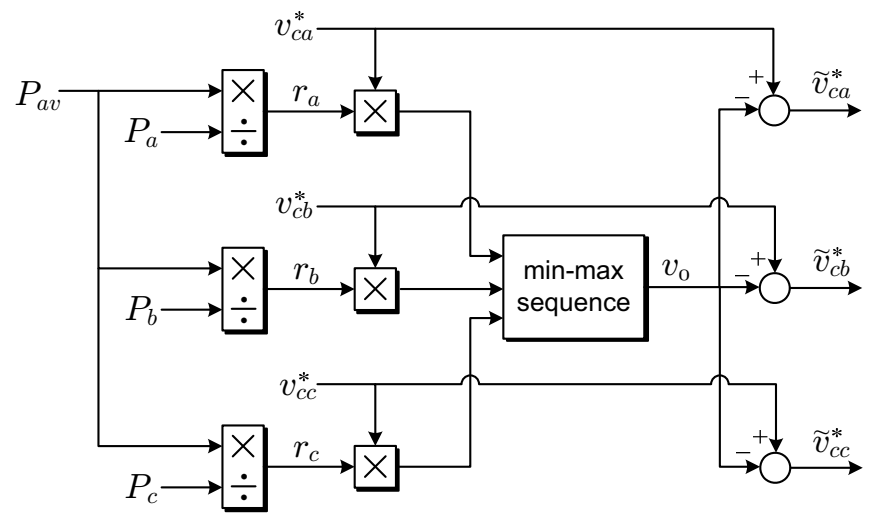

Fig. 4. Proposed phase power imbalance compensation min-max injection block diagram.

It can be seen that the grid currents depend in part on the common mode voltage $v_{n N}$, revealing that common voltage to the three phases can affect the currents. This is an important characteristic because it shows that is possible to balance the currents by moving the neutral point of the converter in a way that the phase voltages are unbalanced inversely proportional to the power unbalance of the converter. This can be easily achieved by computing an imbalance ratio $r_{i}(i=a, b, c)$ for each cell given by

$$
r_{a}=\frac{P_{a v}}{P_{a}}, r_{b}=\frac{P_{a v}}{P_{b}}, r_{c}=\frac{P_{a v}}{P_{c}}
$$

where, $P_{i}(i=a, b, c)$ is the power of each phase and $P_{a v}$ is the average power given by

$$
P_{a v}=\frac{P_{a}+P_{b}+P_{c}}{3}
$$

The power unbalance ratios are multiplied to the corresponding voltage reference $\left(v_{c a}^{*}, v_{c b}^{*}, v_{c c}^{*}\right)$ in per unit, which weights or compensates the amplitude of the references according to the unbalance. Then a min-max zero sequence voltage $v_{0}$ of these weighted references is computed by

$$
\begin{aligned}
v_{0}= & \frac{\max \left\{r_{a} v_{c a}{ }^{*}, r_{b} v_{c b}{ }^{*}, r_{c} v_{c c}{ }^{*}\right\}}{2} \\
+ & \frac{\min \left\{r_{a} v_{c a}{ }^{*}, r_{b} v_{c b}{ }^{*}, r_{c} v_{c c}{ }^{*}\right\}}{2} .
\end{aligned}
$$

and injected to each reference to introduce the corresponding neutral shift. This originates the compensated phase reference voltages

$$
\tilde{v}_{c a}^{*}=v_{c a}^{*}-v_{0}, \tilde{v}_{c b}^{*}=v_{c b}^{*}-v_{0}, \tilde{v}_{c c}^{*}=v_{c c}^{*}-v_{0} .
$$

The block diagram that performs this simple compensation is given in Fig. 4.

The advantage of balancing the currents in this way is that there is no need to implement more sensors or estimators, because the powers are already available as they are needed 

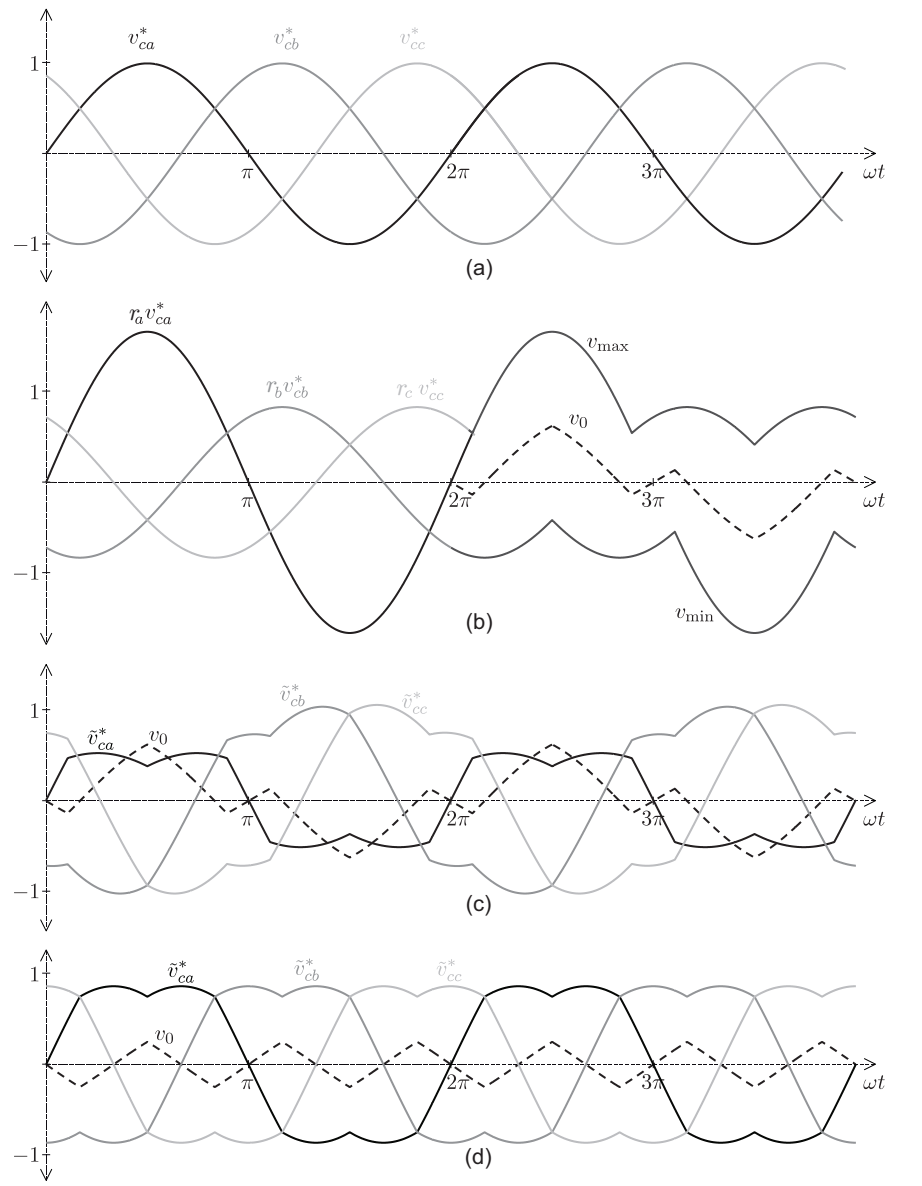

Fig. 5. Example of phase $a$ imbalance reference compensation: a) three-phase converter reference voltages, b) Imbalance compensated reference voltages, c) Imbalance compensated reference voltages with min-max sequence injection, d) Particular case whit no phase imbalance (traditional min-max).

for the MPPT algorithm of the dc-dc converter stage, and furthermore, the computational cost is really low, since it consists of a ratio calculation and zero sequence injection, which is usually included in conventional modulation of commercial converters. In addition it presents a good dynamic behavior as the ratios are computed directly from the power fluctuations in the phases.

To better understand the mechanism, a qualitative example of the original references, the weighted references, the minmax zero sequence and the compensated references for an imbalance in phase $a$ are given in Fig. 5a,b and c respectively. Fig. 5d shows the compensated references in case no unbalance is present, which corresponds to traditional minmax sequence injection.

2) Per-cell imbalance compensation: Once the per-phase imbalance has been compensated, there is still the chance that the power delivered to the different cells of one phase of the converter is also unbalanced. The converter is modulated using phase-shifted PWM (more information on PS-PWM can be found in [17]) and therefore the compensated voltage reference given by the VOC loop is the same for all the cells. The carrier signals are shifted to produce the multilevel stepped waveform,

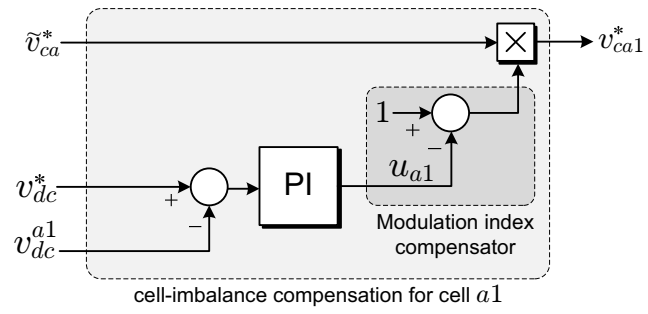

Fig. 6. Per cell power imbalance compensation for PS-PWM (only power cell $a 1$ given as example).

but essentially will impose the same average usage of the cells drawing similar average current from each one. In presence of unbalance this will cause voltage drift of the dc-links causing distortion in the converter voltage and possible damage of the power cells.

This type of imbalance has been dealt with in the past for single phase CHB PV systems. The same approach used in [14] is considered in this work, and therefore will not be addressed in full detail. The idea behind the balancing mechanism is to distribute the usage of the cells of one phase in the same proportion of the imbalance by adjusting (redistributing) the on and off times given by the PWM strategy. In this way, more or less active power is drawn by the cell according to the imbalance experimented by the system. Fig. 6 presents the balancing scheme block diagram given as example for cell $a 1$ of the converter.

The dc-link voltage error $\left(v_{d c}^{*}-v_{d c}^{a 1}\right)$ is regulated with a PI controller, whose output is used to adjust the amplitude of the per unit reference signal. In this way the amplitude of the references used for each cell are modified proportionally to the error of their respective dc-link voltages. This results in a feedforward correction that redistributes the on and off times so that balance is achieved.

\section{RESUlts}

A three-phase 7-level CHB with three cells per phase rated at $3.3 \mathrm{kV}$ is considered for simulation results. Each dc-link is controlled to $1150 \mathrm{~V}$. The PV module modeled and simulated is based on the Sharp/NU-U235F1 module (rated power output of $235 \mathrm{~W}$ and $30 \mathrm{~V}$ rated voltage at $25^{\circ} \mathrm{C}$ with a solar radiation of $1 \mathrm{~kW} / \mathrm{m}^{2}$ ). Considering the dc-link voltage and the module rated output voltage a total of $30 \mathrm{PV}$ modules in series are considered. In addition to have a more realistic simulation in terms of power output, 20 strings, each with individual dc-dc stage have been paralleled per power cell. This makes a total of 5400 modules rated at total of 1.26MW. Matlab/simulink coupled with PSIM has been used as simulation tool. Other parameters of the system and simulation are given in Table I.

To test the dynamic and steady state performance of the proposed imbalance control method, two step changes have been performed to the radiation level. All power cells of the converter start at rated temperature $\left(25^{\circ} \mathrm{C}\right)$ and radiation $\left(1 \mathrm{~kW} / \mathrm{m}^{2}\right)$. At $t=0.5 \mathrm{~s}$ a step change to the radiation of the strings connected to cell $a 2$ and $a 3$ is performed to force a per cell imbalance. Radiation is lowered to $0.75 \mathrm{pu}$ and $0.5 \mathrm{pu}$ 
TABLE I

SIMULATION PARAMETERS

\begin{tabular}{ccc}
\hline \hline Parameter & Symbol & Value \\
\hline Line to Line Grid Voltage & $v_{s_{l l}}$ & $3.3 \mathrm{kV}_{\mathrm{RMS}}$ \\
Grid Frequency & $f_{s}$ & $50 \mathrm{~Hz}$ \\
Rated Power & $P_{\mathrm{nom}}$ & $1.2 \mathrm{MW}$ \\
Rated Current & $i_{s \mathrm{nom}}$ & $300 \mathrm{~A}_{\mathrm{RMS}}$ \\
Input filter inductance & $L_{s}$ & $2 \mathrm{mH}$ \\
Input filter resistance & $R_{s}$ & $0.1 \mathrm{~m} \Omega$ \\
DC-link capacitance & $C$ & $3700 \mu \mathrm{F}$ \\
DC-link voltage reference per cell & $v_{d c}{ }^{*}$ & $1150 \mathrm{~V}$ \\
Total DC-link voltage per phase & $3 v_{d c}{ }^{*}$ & $3450 \mathrm{~V}$ \\
Device avge. switching freq. & $f_{\mathrm{sw}}$ & $500 \mathrm{~Hz}$ \\
Equivalent output freq. per phase & $f_{\mathrm{sw}}$ & $3000 \mathrm{~Hz}$ \\
No. of strings connected to each dc-bus & $N_{p}$ & 20 \\
No. of series connected PV modules per string & $N_{s}$ & 30 \\
Open circuit voltage of module & $V_{\mathrm{oc}}$ & $37 \mathrm{~V}$ \\
Maximum power voltage of module & $V_{\mathrm{pm}}$ & $30 \mathrm{~V}$ \\
Short circuit current of module & $V_{\mathrm{sc}}$ & $8.6 \mathrm{~A}$ \\
Maximum power current of module & $I_{\mathrm{pm}}$ & $7.84 \mathrm{~A}$ \\
\hline \hline
\end{tabular}

respectively. This is an extremely high imbalance of up to $50 \%$ compared to cell $a 1$. Note that this imbalance also implies a per phase imbalance since, phase $a$ of the converter will be operating at lower power than phase $b$ and $c$. In addition, a harder per phase imbalance is forced at $t=0.6 \mathrm{~s}$ by reducing the radiation to all the strings connected to phase $c$ in 50\%. Phase $b$ is the only one not modified throughout the experiment. The simulation results for the dynamic changes described earlier are illustrated in Fig. 7-9.

The effect of the power imbalances can be better appreciated in the change introduced to the voltage reference signals, shown in Fig. 7. In Fig. 7a the phase compensated voltage references are illustrated. It can be clearly appreciated that during balanced operation up to $0.5 \mathrm{~s}$ no compensation is performed obtaining the traditional min-max injection reference waveform studied earlier in Fig. 5d. From $0.5 \mathrm{~s}$ to $0.6 \mathrm{~s}$ there is a small per phase imbalance due to the changes introduced to phase $a$. This can be seen in Fig. 7a, where the weighted minmax compensation becomes evident for phases $b$ and $c$. The per cell imbalance in phase $a$ is noticeable in Fig. 7b where the amplitudes of the references for each cell of phase $a$ are modified according to the respective imbalance. Note how the amplitude of reference of cell $a 3$ is reduced almost to the half due to the $0.5 \mathrm{pu}$ radiation step change. By reducing the voltage reference, the on times are shorter reducing the power drawn from the cell, enabling the dc-link voltage to stay at the desired level. Finally, at $t=0.6 \mathrm{~s}$ the per phase imbalance becomes stronger since all the strings of phase $c$ have experienced a $50 \%$ reduction in radiation. Note that per-phase and per-cell imbalances are occurring at the same moment after $t=0.6 \mathrm{~s}$. This is not a problem, since both compensations are performed sequentially one after the other allowing to control both type of imbalances.

The overall performance of the VOC can be observed

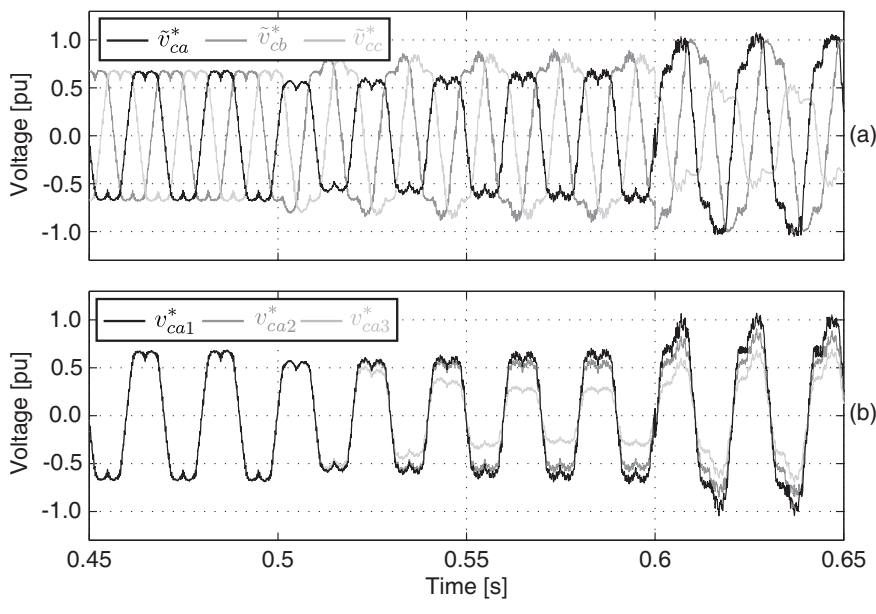

Fig. 7. Converter reference voltages: a) Compensated references $\left(\tilde{v}_{c a}^{*}, \tilde{v}_{c b}^{*}\right.$, $\tilde{v}_{c c}^{*}$ ) for a phase imbalance in phase $c$ at $\left.t=0.6[\mathrm{~s}], \mathrm{b}\right)$ Compensated references for each cell of phase $a\left(v_{c a 1}^{*}, v_{c a 2}^{*}, v_{c a 3}^{*}\right)$ with cell imbalance at $t=0.5[\mathrm{~s}]$.

in Fig. 8. The resulting modulated converter output voltage waveforms of the references analyzed previously are shown in Fig. 8a. The imbalance compensation and min-max sequence can be clearly appreciated in these inverter phase voltages. Since the min-max injection is a zero sequence it should not appear in the line-line voltages, this can be appreciated in Fig. 8b, which appears sinusoidal in shape. The accurate performance of the imbalance compensation can be better appreciated in Fig. 8c, where the three-phase grid currents appear completely balanced, despite the converter phases are operating at different power levels. The grid phase voltage $v_{s a}$ has been scaled down and plotted together with the currents (dashed line) to show the proper synchronism achieved with the PLL.

The power processed by each cell of phase $a$ of the converter is illustrated in Fig. 8d. Here the effect of the step change in the solar radiation given to cell $a 2$ and $a 3$ is clearly noticeable. Finally the total power of each phase of the converter are given in Fig. 8e. Apart from the power reduction at $t=0.5 \mathrm{~s}$ of phase $a$ the step change in radiation in phase $c$ at $t=0.6 \mathrm{~s}$ can also be appreciated. Note that phase $b$ does not experiences significant power changes throughout the experiment, confirming that the different dc-dc stages and multistring PV systems operate independently from each other at their own MPPT. The system proves to keep working with high performance and power quality despite the severe imbalances introduced to the operation of the different cells and phases. The step changes introduced in this experiment are very challenging and more extreme than real conditions, since module mismatch and partial shading of $50 \%$ in a large scale PV plants are not common.

To fully validate the imbalance compensation control method the dc-link voltages of the three cells of each phase are given in Fig. 9. The dynamic behavior of the per-cell imbalance compensation can be appreciated in the dc-link voltages of phase $a$ shown in Fig. $9 \mathrm{a}$, from $t=0.5 \mathrm{~s}$. Although 

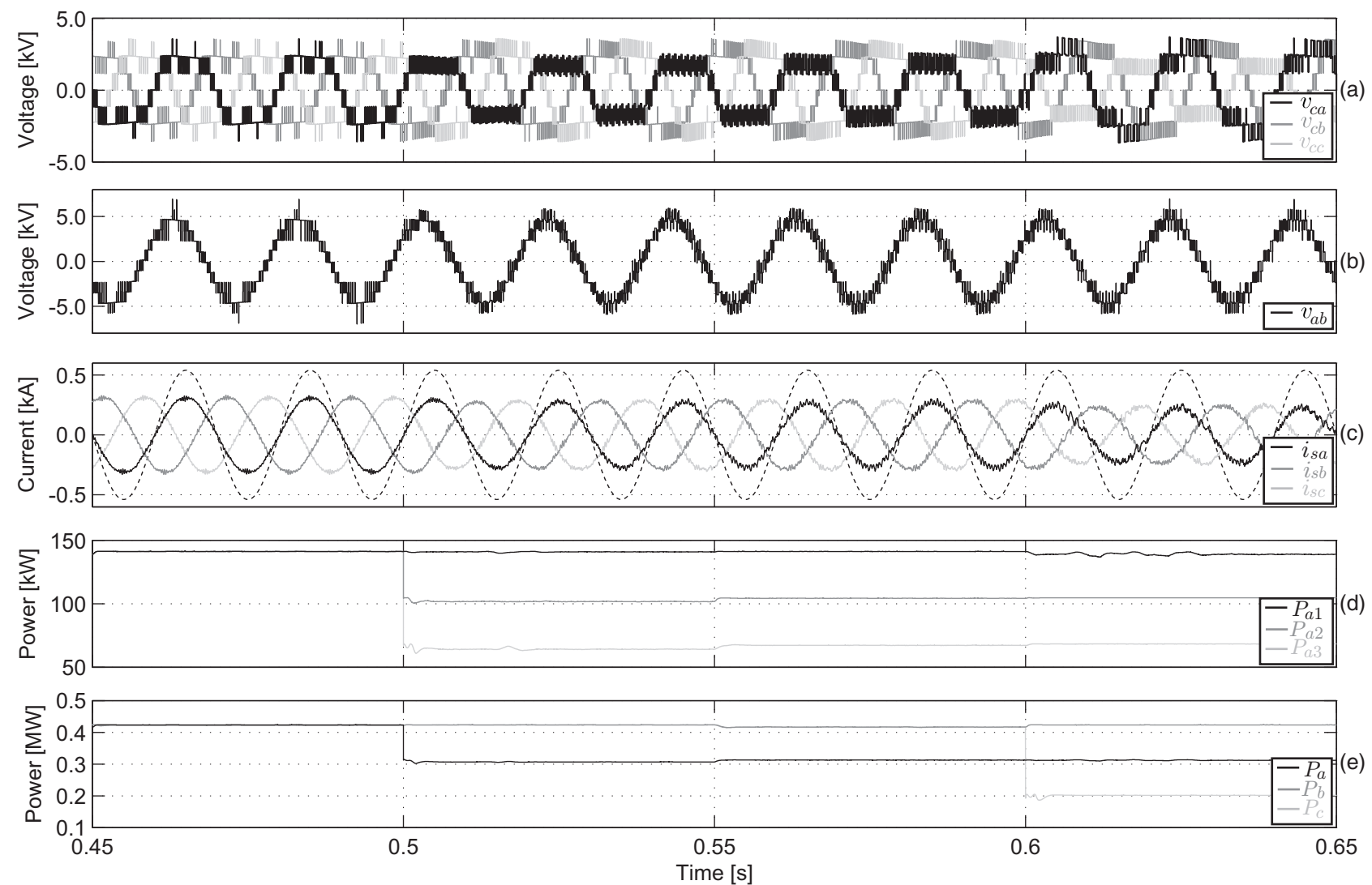

Fig. 8. Dynamic performance with a step cell imbalance in phase $a$ at $t=0.5[\mathrm{~s}]$ and a step phase imbalance in phase $c$ at $t=0.6[\mathrm{~s}]$ : a) Converter phase voltages $\left(v_{c a}, v_{c b}, v_{c c}\right)$, b) Line-line converter voltage $\left.\left(v_{a b}\right), \mathrm{c}\right)$ Grid currents $\left(i_{s a}, i_{s b}, i_{s c}\right)$ with grid voltage $v_{s a}$ shown scaled down to highlight synchronism, d) Power processed by each cell of phase $s\left(P_{a 1}, P_{a 2}, P_{a 3}\right)$, e) Power processed by each phase $\left(P_{a}, P_{b}, P_{c}\right)$.
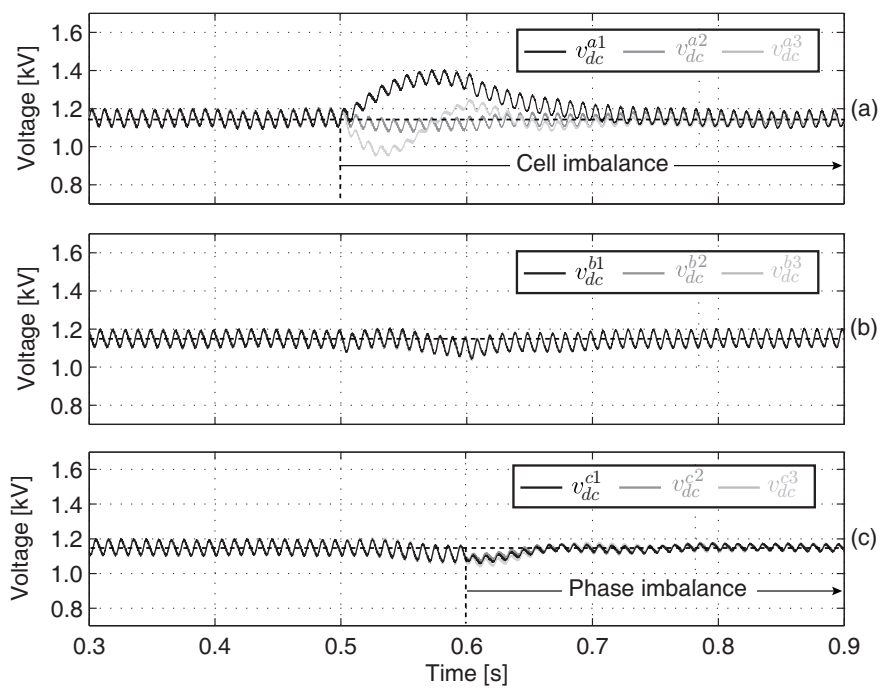

Fig. 9. Dc-link voltages with cell imbalance in phase $a$ at $t=0.5[\mathrm{~s}]$ and with phase imbalance in phase $c$ at $t=0.6[\mathrm{~s}]$ : a) of phase $a\left(v_{d c}^{a 1}, v_{d c}^{a 2}, v_{d c}^{a 3}\right)$, b) of phase $\left.b\left(v_{d c}^{b 1}, v_{d c}^{b 2}, v_{d c}^{b 3}\right), \mathrm{c}\right)$ of phase $c\left(v_{d c}^{c 1}, v_{d c}^{c 2}, v_{d c}^{c 3}\right)$.

the compensation for the per-phase imbalance applied to phase $c$ is achieved compensating the three phases, the impact on the dc-link voltages of phase $a$ and $b$ is negligible. In fact, the dc-link voltages of phase $b$ which has not experimented any change in radiation presents very small deviation from the reference due to minimal coupling of the variables.

The simulation results show that the proposed method can achieve the control goals meeting the grid side requirements (power quality) and the PV system maximum power output for the given radiation.

\section{CONCLUSION}

A new medium voltage converter interface for large scale PV energy conversion systems is presented. It is based on a three-phase CHB multilevel multistring topology. The multistring converter structure composed of isolated dc-dc and a grid tied dc-ac converter, effectively decouples the grid side control form the PV strings control requirements. This allows independent MPPT control of each string without affecting the dc-link voltages of each cell.

The main challenges related to the proposed configuration are the possible existence of two types of power imbalance: between the power cell of one phase of the converter and between the phases of the converter. These challenges are solved by including two simple feedforward compensations: one applied to the reference voltage of each phase by means of a power ratio and a min-max zero sequence, and another by adjusting the modulation index of the different references of each cell used in the phase-shifted modulation of a phase of 
the converter. The proposed compensation methods can work even under combined power imbalances.

The three-phase CHB multistring topology with the proposed control and imbalance compensation methods, enables to concentrate in a single medium voltage converter a large scale PV plant of up to 120 MVA. Additional advantages are the inherent superior power quality of the CHB (compatible with current grid codes), low switching frequency (higher efficiency), medium voltage grid connection and possible fault tolerant operation.

\section{ACKNOWLEDGMENT}

The authors gratefully acknowledge financial support provided by Fondecyt (no. 1110783), by Centro CientíficoTecnológico de Valparaíso (CCTVal) $\mathrm{N}^{\circ}$ FB021 of Universidad Técnica Federico Santa María, and by the Spanish Ministry of Education under grant PR2010-0162.

\section{REFERENCES}

[1] Renewable Energy Policy Network for the 21st Century, "Renewables 2010 global status report," available at http://www.ren21.net/publications, 2010.

[2] pvresources.com, "Large-scale photovoltaic power plants: ranking," available at http://www.pvresources.com/en/top50pv.php.

[3] F. Blaabjerg, Z. Chen, and S. B. Kjaer, "Power electronics as efficient interface in dispersed power generation systems," IEEE Trans. Power Electron., vol. 19, no. 5, pp. 1184-1194, Sep. 2004.

[4] M. Meinhardt and G. Cramer, "Multi-string-converter: The next step in evolution of string-converter technology," in in Proc. 9th Eur. Power Electronics and Applications Conf., 2001.

[5] S. B. Kjaer, J. K. Pedersen, and F. Blaabjerg, "A review of single-phase grid-connected inverters for photovoltaic modules," IEEE Trans. Ind. Applicat., vol. 41, no. 5, pp. 1292-1306, Sep./Oct. 2005.

[6] "Grid code - high and extra high voltage," E.ON Netz Gmbh, April 2006

[7] M. Calais and V. G. Agelidis, "Multilevel converters for single-phase grid connected photovoltaic systems-an overview," in Industrial Electronics, 1998. Proceedings. ISIE '98. IEEE International Symposium on, vol. 1, Jul. 7-10, 1998, pp. 224-229.

[8] S. Busquets-Monge, J. Rocabert, P. Rodriguez, S. Alepuz, and J. Bordonau, "Multilevel Diode-clamped Converter for Photovoltaic Generators With Independent Voltage Control of Each Solar Array," IEEE Trans. Ind. Electron., vol. 55, no. 7, pp. 2713-2723, Jul. 2008.
[9] J. Selvaraj and N. A. Rahim, "Multilevel Inverter For Grid-connected PV System Employing Digital PI Controller," IEEE Trans. Ind. Electron., vol. 56, no. 1, pp. 149-158, Jan. 2009.

[10] N. A. Rahim and J. Selvaraj, "Multi-string Five-level Inverter with Novel PWM Control Scheme for PV Application," IEEE Transactions on : Accepted for future publication Industrial Electronics, pp. 1-1, / 2009.

[11] G. Grandi, C. Rossi, D. Ostojic, and D. Casadei, "A New Multilevel Conversion Structure for Grid-connected PV Applications," IEEE Trans. Ind. Electron., vol. 56, no. 11, pp. 4416-4426, Nov. 2009.

[12] E. Villanueva, P. Correa, J. Rodriguez, and M. Pacas, "Control of a Single-phase Cascaded H-bridge Multilevel Inverter for Grid-connected Photovoltaic Systems," IEEE Trans. Ind. Electron., vol. 56, no. 11, pp. 4399-4406, Nov. 2009.

[13] L. Ma, X. Jin, T. Kerekes, M. Liserre, R. Teodorescu, and P. Rodriguez, "The PWM strategies of grid-connected distributed generation active NPC inverters," in Energy Conversion Congress and Exposition, 2009. ECCE. IEEE, Sep. 20-24, 2009, pp. 920-927.

[14] S. Kouro, A. Moya, E. Villanueva, P. Correa, B. Wu, and J. Rodriguez, "Control of a cascaded h-bridge multilevel converter for grid connection of photovoltaic systems," in 35th Annual Conference of the IEEE Industrial Electronics Society (IECONO9), 2009, pp. 1-7.

[15] S. Kouro, K. Asfaw, R. Goldman, R. Snow, B. Wu, and J. Rodríguez, "Npc multilevel multistring topology for large scale grid connected photovoltaic systems," in 2010 2nd IEEE International Symposium on Power Electronics for Distributed Generation Systems (PEDG 2010), 2010, pp. 400-4005.

[16] S. Kouro, M. Malinowski, K. Gopakumar, J. Pou, L. G. Franquelo, B. Wu, J. Rodriguez, M. A. Pérez, and J. I. Leon, "Recent advances and industrial applications of multilevel converters," IEEE Trans. Ind. Electron., vol. 57, no. 8, pp. 2553-2580, August 2010.

[17] J. Rodriguez, L. G. Franquelo, S. Kouro, J. I. Leon, R. C. Portillo, M. A. M. Prats, and M. A. Perez, "Multilevel Converters: An Enabling Technology for High-power Applications," Proc. IEEE, vol. 97, no. 11, pp. 1786-1817, Nov. 2009.

[18] D. P. Hohm and M. E. Ropp, "Comparative study of maximum power point tracking algorithms," Progress in Photovoltaics: Research and Applications, vol. 11, no. 1, pp. 47-62, 2003.

[19] N. Femia, D. Granozio, G. Petrone, G. Spagnuolo, and M. Vitelli, "Predictive \& adaptive mppt perturb and observe method," IEEE Transactions on Aerospace and Electronic Systems, vol. 43, no. 3, pp. 934 950, 2007.

[20] M. Malinowski, M. P. Kazmierkowski, and A. M. Trzynadlowski, "A comparative study of control techniques for pwm rectifiers in ac adjustable speed drives," IEEE Transactions on Power Electronics, vol. 18 no. 6, pp. 1390-1396, November 2003. 